\title{
XCIII. On the gas-equation
}

\section{S.D. Wicksell}

To cite this article: S.D. Wicksell (1912) XCIII. On the gas-equation, Philosophical Magazine Series 6, 24:144, 869-876, DOI: 10.1080/14786441208634881

To link to this article: http://dx.doi.org/10.1080/14786441208634881

曲 Published online: 20 Apr 2009.

Submit your article to this journal 주다

Џll Article views: 2

Q View related articles $₫$ 
side. Case (ii.) is thus deducible from case (i.) by the application of a smoothing process to $\mathrm{C}$ and $\mathrm{S}$, whereby fluctuations of small length are removed.

We may sum up by saying that a smoothing of $\phi(x)$ annuls $C$ and $S$ for large values of $u$, while a smoothing of $\mathrm{C}$ and $\mathrm{S}$ (as functions of $u$ ) annuls $\phi(x)$ for values of $x$ which are numerically great.

Terling Place, Withnm, October 1912.

XCIII. On the Gas-equation. $B y$ S. D. WiCKseli, Lund, Siceden *.

J the famous gas-equation of yan der Waals :

$$
\left(\mathrm{P}+\frac{a}{v^{2}}\right)(v-b)=\mathrm{R} \theta, \ldots . \quad . \quad .
$$

$a$ and $b$ are small constants depending, $a$ on the mutual attraction of the molecules, and $b$ on the volume of the molecules.

$\dot{P}$ is the pressure of the gas, $v$ its volume, and $\theta$ denotes the absolute temperature. $\mathrm{R}$ is the gas-constant.

In reality, however, the quantities $a$ and $b$ are not constant. They vary both with temperature and pressure.

In the following I will regard $a$ and $b$ as functions of the temperature only; thus getting a gas-equation that covers the case of Clausius and several others.

It is the object of this paper to transform the gas-equation into a more convenient form by means of series, and to study the quantities $a$ and $b$, expressing them in known and measurable quantities.

The equation (1) can be transformed into

$$
\mathrm{P} v\left(1-\frac{b}{v}\right)=\mathrm{R} \theta\left[1-\frac{a}{v \operatorname{R} \theta}\left(1-\frac{b}{v}\right)\right] .
$$

We express $\mathbf{P}$ in atmospheres;

$v$ in the volume of one gram-molecule of the ideal gas at on $\theta$ atmosphere and $0^{\circ}$ centigrade;

$\theta$ in degrees centigrade.

By index zero $I$ mean the value at $0^{\circ} \mathrm{C}$.

- Communicated by the Author. 
We then have

and putting

$$
\mathrm{R}=\frac{1}{\bar{\theta}_{0}},
$$

$$
\begin{aligned}
a \frac{\theta_{0}}{\bar{\theta}} & =\alpha, \\
1 / v & =x, \\
\mathrm{P} \cdot \frac{\theta_{0}}{\theta} & =y,
\end{aligned}
$$

we get

$$
y(1-b x)=x-\alpha x^{2}+\alpha b x^{3} . \quad \cdot \quad \cdot .
$$

I now want $x$ expressed as a series in powers of $y$ or, which is the same, in powers of $P$.

The first question is how and when this series is convergent.

The equation (2) gives

$$
x=\mathrm{F}(y),
$$

and the series is convergent for all values of $y$ less than the absolute value of the least $y$ that gives $\mathrm{F}(y)$ a singularity.

It is easily seen that $\mathrm{F}(y)$ can have no other finite singularity than when it ramifies, and that occurs when $y$ has such $a$ value that the cubic (2) has equal roots. For every value of the parameters $a$ and $b$, that is of the temperature, there are three such values of $y$, always one negative and two positive or imaginary. They are given by the equation

$$
4 b^{4} y^{3}+y^{2}\left(12 b^{3}+8 a b^{2}\right)-y\left(20 a b-12 b^{2}-4 \alpha^{2}\right)+4 b-a=0 .
$$

It is seen from this equation that when $\alpha=4 b$ or $\theta=\frac{a}{4 b} \theta_{0}$ one of the roots is equal to zero. The least absolute value of the roots of the equation is the interval of convergence. For $\theta=\frac{a}{4 b} \theta_{0}$ this interval must be equal to zero. It is also easy to see that when $\theta$ grows the equation has two positive roots and one negative, which always grow until $\theta=\frac{8 a}{27 b} \theta_{0}$, when the positive roots coincide and then turn imaginary. At $\theta=\frac{8 a}{27 b} \theta_{0}$ the interval of convergence is $|y|=\frac{1}{8 b}$. $\theta=\frac{8 a}{27 b} \theta_{0}$ is, theoretically from van der Waals's equation, 
the critical temperature. At $\theta=\infty$ the interval of convergence will be $|y|=\frac{1}{b}$.

The series in powers of $\mathrm{P}$ has an interval of convergence

$$
\mathrm{P}_{c}=\frac{a}{27 \bar{b}^{2}} \delta
$$

where $\delta=0$ for $\theta=\frac{a}{4} \bar{b} \theta_{0}$; and then grows with $\theta$ and becomes equal to 1 for $\theta=\frac{8 a}{27 b} \theta_{0}$; thereafter $\delta$ grows infinitely with $\theta$.

It will be seen from numerical examples that the series generally converges very fast.

In order to find the series we put

$$
x=a_{1} y+a_{2} y^{2}+a_{3} y^{3}+\ldots
$$

and substitute this in the equation (2).

We then get

$$
\begin{aligned}
y & =a_{1} y+a_{2} y^{2}+a_{3} y^{3}+a_{4} y^{4}+a_{5} y^{5}+\ldots \ldots \\
& +b\left(a_{1} y^{2}+a_{2} y^{3}+a_{3} y^{4}+a_{4} y^{5}+\ldots \ldots\right) \\
& -\alpha\left[a_{1}^{2} y^{2}+2 a_{1} a_{2} y^{3}+\left(a_{2}^{2}+2 a_{1} a_{3}\right) y^{4}+\left(2 a_{1} a_{4}+2 a_{2} a_{3}\right) y^{5}+\ldots\right] \\
& +b \alpha\left[a_{1}^{3} y^{3}+3 a_{1}^{2} a_{2} y^{4}+3\left(a_{1}^{2} a_{3}+a_{2}{ }^{2} a_{1}\right) y^{5}+\ldots . .\right.
\end{aligned}
$$

As $\alpha$ and $b$ are supposed independent of $\mathrm{P}$, or which is the same, of $y$, we can put the coefficients of the powers of $y$ equal to zero.

That gives us

$$
\begin{aligned}
& a_{1}=1, \\
& a_{2}=\alpha-b, \\
& a_{3}=2 \alpha a_{2}-b a_{2}-b \alpha, \\
& a_{4}=\alpha\left(a_{2}+2 a_{3}\right)-b a_{3}-3 b \alpha a_{2}, \\
& a_{5}=2 \alpha\left(a_{4}+a_{2} a_{3}\right)-b a_{4}-3 b \alpha\left(a_{3}+a_{2}^{2}\right) .
\end{aligned}
$$

Putting $\alpha-b=\phi$ and solving the system we get

$$
\begin{aligned}
& a_{1}=1, \\
& a_{2}=\phi, 1 \\
& a_{3}=2 \phi^{2}-b^{2}, \\
& a_{4}=5 \phi^{3}-5 b^{2} \phi-b^{3} \\
& a_{5}=14 \phi^{4}-21 \phi^{2} b^{2}-6 b^{3} \phi+2 b^{4} .
\end{aligned}
$$


And finally we get

$$
\begin{aligned}
x=y & {\left[1+\phi y+\left(2 \phi^{2}-l^{2}\right) y^{2}+\left(5 \phi^{3}-5 b^{2} \phi-b^{3}\right) y^{3}\right.} \\
& \left.+\left(14 \phi^{4}-21 \phi^{2} b^{2}-6 b^{3} \phi+2 b^{4}\right) y^{4}+\ldots\right] . .
\end{aligned}
$$

For the ideal gas we have

$$
y=x,
$$

and we see that the series $1+\phi y+\ldots$ can, according to the nature of the constants, have no value equal to zero. Accordingly, we have a right to invert the series, and thus we get

$$
\begin{array}{r}
\mathrm{P} v=\frac{\theta}{\theta_{0}}\left[1-\frac{\theta_{0}}{\theta^{-}} \phi \mathrm{P}-\left(\begin{array}{c}
\theta_{0} \\
\dot{\theta}
\end{array}\right)^{2}\left(\phi^{2}-b^{2}\right) \mathrm{P}^{2}-\left(\frac{\theta_{0}}{\theta}\right)^{3}\left(2 \phi^{3}-3 \phi b^{2}-b^{3}\right) \mathrm{P}^{3}\right. \\
\left.-\left(\begin{array}{c}
\theta_{0} \\
\theta
\end{array}\right)^{4}\left(5 \phi^{4}-10 \phi^{2} b^{2}-4 \phi b^{3}+b^{4}\right) \mathrm{P}^{4}-\ldots\right]
\end{array}
$$

The following numerical examples will illustrate the magnitude of the different terms.

For carbon-dioxide we take at $0^{\circ} \mathrm{C}$.,

$$
\begin{aligned}
& a=0.011 \\
& b=0.003 .
\end{aligned}
$$

(All values are, when nothing else is stated, taken from the work Die Zustandsgleichung by Prof. Dr. Kuenen.)

Then (4) becomes

$$
\mathrm{P} v=1-0.008 \mathrm{P}-0.00005 \mathrm{P}^{2}-0.000003 \mathrm{P}^{3} \text {. }
$$

For ethylene we take at $20^{\circ} \mathrm{C}$.

and get

$$
\begin{aligned}
& a=0.00786 \\
& b=0.0024,
\end{aligned}
$$

$$
\mathrm{P}_{v}=1.073-0.0050 \mathrm{P}-0.000017 p^{2}-0.00000011 \mathrm{P}^{3} \text {. }
$$

The results are given by the following table :-

$\begin{array}{cccccc}\mathbf{P} . & \mathbf{P} 0.0050 . & \mathbf{P}^{2} 0.000017 . & \mathbf{P}^{3} 0.00000011 . & \mathbf{P} v . & \mathbf{P} v . \text { from (1). } \\ 31.9 & 0.16 & 0.02 & 0.003 & 0.89 & 0.89 \\ 45.8 & 0.22 & 0.04 & 0.01 & 0.80 & 0.78\end{array}$

It is seen that at 30 atmospheres we need not go farther than to the second power of $\mathrm{P}$ in order to obtain a value of $\mathrm{P} v$ with two decimals. It is furthermore seen that for relatively low pressures, generally as high as $\tilde{\mathbf{a}}$ atm., the second power of $\mathrm{P}$ can be neglected, if we want $\mathrm{P} v$ given with three decimals. 
We then have

$$
\begin{array}{ll} 
& \mathrm{P} v=\frac{\theta}{\theta_{0}}-\phi \mathrm{P}, \ldots . \\
\text { or } & \mathrm{P} v-\mathrm{R} \theta=\phi \mathrm{P} .
\end{array}
$$

That is, the deviation from the ideal gas-equation is proportional to the pressure. This relation can be written

$$
\frac{\mathrm{P}^{\prime} v^{\prime}-\mathrm{R} \theta}{\mathrm{P}^{\prime \prime} v^{\prime \prime}-\mathrm{R} \theta}=\frac{\mathrm{P}^{\prime}}{\mathrm{P}^{\prime \prime}}
$$

where $\mathrm{P}^{\prime}$ and $v^{\prime}$ are referred to one case and $\mathrm{P}^{\prime \prime}$ and $v^{\prime \prime}$ to another at the same temperature.

The equation (6) includes no quantity characteristic for the gas, and therefore can be regarded as a general gas-law in the second approximation if Boyle-Mariotte-Gay Lussac's law is regarded as the first approximation.

It will be of some interest to see what form the expression of the expansion coefficient $k^{\mathrm{P}}$ will take as a series in powers of $P$ and $\theta$.

We have

and get from (4)

$$
\frac{d v}{v_{0}}=k^{\mathbf{P}} d \theta
$$

$$
\begin{array}{r}
\mathrm{P} d v=\frac{d \theta}{\theta_{0}}\left[1+\mathrm{P}(\phi+b) \frac{\theta_{0}}{\theta}+\mathrm{P}^{2}\left(3 \phi^{2}+2 \phi b-b^{2}\right)\left(\frac{\theta_{0}}{\theta}\right)^{2}\right. \\
\left.+\mathrm{P}^{3}\left(10 \phi^{3}-9 \phi b^{2}+6 \phi^{2} b-4 b^{3}\right)+\ldots\right] .
\end{array}
$$

From (3) we get

$$
\frac{1}{\mathrm{P} v_{0}}=1+\mathrm{P} \phi_{0}+\mathrm{P}^{2}\left(2 \phi_{0}{ }^{2}-b^{2}\right)+\mathrm{P}^{3}\left(5 \phi_{0}{ }^{3}-5 \phi_{0} b^{2}-b^{3}\right)+\ldots
$$

As

$$
\phi_{0}=a-b \text {, }
$$

we finally get

$$
\begin{aligned}
& \begin{array}{r}
k^{\mathrm{P}}=\frac{1}{\theta_{0}}\left[1+\mathrm{P}\left(\frac{\theta_{0}^{2}+\theta^{2}}{\theta^{2}} a-b\right)+\mathrm{P}^{2}\left(\frac{3 \theta_{0}^{4}+\theta_{0}{ }^{2} \theta^{2}+2 \theta^{4}}{\theta^{4}} a\right.\right. \\
\left.\left.-\frac{4 \theta_{0}{ }^{3}+\theta_{0}{ }^{2} \theta+4 \theta^{3}}{\theta^{3}} a b+b^{2}\right) \ldots\right]
\end{array} \\
& \text { or if } \quad \begin{array}{r}
\mathrm{P} \frac{\theta_{0}}{\theta}=y, \quad a \frac{1}{\theta \theta_{0}}=\beta, \quad \frac{b}{\theta_{0}}=\gamma, \\
k^{\mathrm{P}}=1 / \theta_{0}\left[1+y\left\{\left(\theta_{0}{ }^{2}+\theta^{2}\right) \beta-\theta_{\gamma}\right\}+y^{2}\left\{\left(3 \theta_{0}{ }^{4}+\theta_{0}{ }^{2} \theta^{2}+2 \theta^{4}\right) \beta^{2}\right.\right. \\
\end{array}
\end{aligned}
$$


The convergence of this series I have not ascertained otherwise than numerically. It is clear, however, that the series cannot converge for a value of $\mathrm{P}$ or $y$ greater than the interval of convergence of ( 3 ) when $\theta=\theta_{0}$.

For not too high pressures we can as before neglect all but the first power in $\mathrm{P}$ and get

$$
k^{\mathrm{P}}=1 / \theta_{0}\left[1+\mathrm{P}\left(\frac{\theta_{0}^{2}+\theta^{2}}{\theta^{2}} a-b\right)\right] . .
$$

That is, if $a$ and $b$ do not vary with $\mathrm{P}$ we have by unvarying temperature:

$$
\mathrm{A}=\frac{k_{\theta}^{\mathbf{P}_{1}} \theta_{0}-1}{k_{\theta}^{\mathbf{P}_{2}} \theta_{0}-1}=\frac{\mathrm{P}_{1}}{\mathrm{P}_{2}}, \ldots . . . . .
$$

where $k_{\theta}^{\mathrm{P}}$ is the expansion-coefficient at constant pressure by a temperature equal to $\theta$ and a pressure equal to $P$.

This formula is similar to $\left(b^{*}\right)$.

I have tested this formula numerically, and the results are given by the following table.

I have used values of $k$ given in the tables of LandoltBörnstein. Those values are not, however, referred to a constant temperature. They denote the mean expansion for an interval of temperature :-

For Carbon dioxide.

I.

Temp.-interval $\left(0^{\circ}-20^{\circ}\right.$ C.). $\quad$ Pressure about 1-2 atm.

$k$.

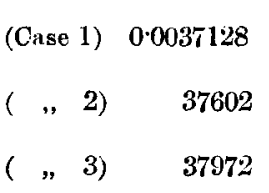

P.

case 1 - $\overline{\boldsymbol{P}}_{2}$

A. $\quad \overline{\mathbf{P}}_{1}$.

$998 \quad \frac{\text { case } 2}{\text { case } 3} \quad 0.376 \quad 0.376$

II.

$\begin{array}{lll}\text { case } 1 & 0 \cdot 727 & 0 \cdot 725\end{array}$

Temp.-interval $\left(64^{\circ}-100^{\circ}\right)$. Pressure about $17-40$ atm.

k. $\quad P$.

(Case 1) $0.004747 \quad 12988 \mathrm{~mm} . \mathrm{Hg}$.

$\left(\begin{array}{llll}\prime 2 & 5435 & 18856\end{array}\right.$

( "3) $6574 \quad 26212$
A.

$\mathrm{P}_{1}$.

case 1

$0.61 \quad 0.68$

$\begin{array}{lll}\text { case } 2 & 0.37 & 0.49\end{array}$

$\begin{array}{lll}\text { case } 1 & 0.60 & 0.71\end{array}$

In this case the pressure is too high to allow us to neglect the second power of $P$. 
For Air.

III.

Temp.-interval unknown.

$k$.

(Case 1) 0.0036706

( , 2) $36944 \quad 2525$

$\left(\begin{array}{lll}\text { ("3) } & 36964 & 2620\end{array}\right.$
P.

$700 \mathrm{~mm} . \mathrm{Hg}$.

Pressure 1-4 atm.

In cases I. and III. the convergence of (8) is evident.

From equations (5) and (7) we evidently can determine $a$ and $b$ in terms of known and measurable quantities.

We have :-

and we get

$$
\begin{aligned}
& \theta \\
& \theta_{0}-\mathrm{P} v=\mathrm{P}\left(a \frac{\theta_{0}}{\theta}-b\right) \\
& k^{\mathrm{P}}-1=\mathrm{P}\left(a \frac{\theta_{0}^{2}+\theta^{2}}{\theta^{2}}-b\right)
\end{aligned}
$$

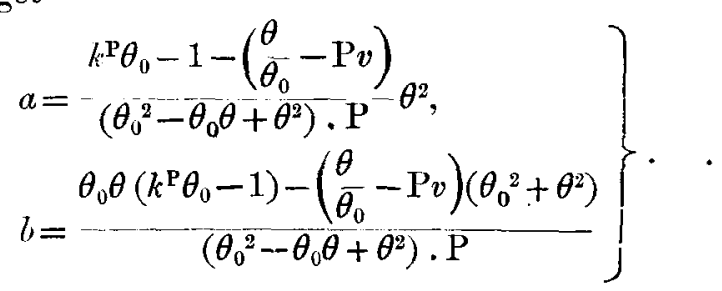

According to van der Waals those expressions ought not to vary. If we regard $a$ and $b$ as constant we can take the case of $0^{\circ} \mathrm{C}$. when $\theta=\theta_{0}$, and get

$$
\left.\begin{array}{l}
a=k_{0}{ }^{\mathrm{P}} \theta_{0}-1-\left(1-v_{0}{ }^{\prime}\right) \\
b=k_{0} \mathrm{P} \theta_{0}-1-2\left(1-v_{0}^{\prime}\right)
\end{array}\right\}
$$

where $v_{0}^{\prime}$ is the volume of one gram-molecule of the gas at $\theta^{\circ}$ and 1 atmosphere, and $a$ and $b$ consequently referred to one gram-molecule of gas.

From (10) we get

$$
a-b=1-v_{0}^{\prime}
$$

That $a-b$ should be expressed in a similar way can a priori be regarded as probable, because the influence of $a$ tends to diminish the volume $v_{0}^{\prime}$ and the influence of $b$ to expand it. 
That this expression is practically true we can see from the following example.

We choose hydrogen as the ideal gas and get the normal volume $v_{1}=22.4452$ litres, which is a little too great. The volume of one gram-molecule of carbon dioxide at $0^{\circ}$ C. and 1 atmosphere is $v_{2}=22 \cdot 2635$ litres.

$$
\begin{gathered}
v_{0}{ }^{\prime} \text { for carbon dioxide is equal to } \frac{v_{2}}{v_{1}} . \\
\text { Consequently } \quad 1-v_{0}{ }^{\prime}=0.0081, \\
a=0.0134, b=0.0054, \quad a-b=0.0080 .
\end{gathered}
$$

From the equation (9) it ought to be possible by trial to determine how $a$ and $b$ vary with temperature at constant pressure, at least when that pressure is not too high, because all the terms in the formula are measurable quantities.

From these lines it is furthermore seen that a series of the form (4) is identically the same as an equation of the form (1), $a$ and $b$ being any function of the temperature. It is evident that from a series in powers of $P$ it ought to be easy to determine by trial what kind of functions of the temperatare the coefficients are, thus getting the variation of $a$ and $b$ by aid of equation (4) for even higher pressures, at least inasmuch as the form of van der Waals' equation is true.

Furthermore, a series of the form (4) is more convenient to use by determining the volume than the solution of the cubic (1).

XCIV. The Effect of ithe Transition Layer of a Liquid on its Surface Tension. By R. D. KLEEMAN, D.Sc., B.A.*

TT requires the expenditure of energy to produce an 1 increase in the area of surface of a liquid. . The surfacetension is therefore the work done, keeping the temperature constant, in producing unit increase in the area of surface of the liquid. Now this increase of surface may be produced in $a$ variety of ways, since it follows from thermodynamics that the work done during an isothermal process between given limits is independent of the nature of the path of the process. One way suggested by Laplace is to suppose a slab of liquid cut into two parts by an imaginary plane, and the parts then separated by an infinite distance from one another. If $\mathrm{W}$ denote the total amount of work done during

* Communicated by the Author. 